\title{
MobileMAN: Mobile Metropolitan Ad Hoc Networks ${ }^{\star}$
}

\author{
Marco Conti ${ }^{1}$, Silvia Giordano ${ }^{2}$, Gaia Maselli ${ }^{1}$, and Giovanni Turi ${ }^{1}$ \\ 1 IIT Institute, CNR, Via G. Moruzzi 1, 56124 Pisa, Italy \\ \{marco.conti, gaia.maselli,giovanni.turi\}@iit.cnr.it \\ 2 SUPSI, Switzerland $(\mathrm{CH})$ \\ silvia.giordano@die.supsi.ch
}

\begin{abstract}
MobileMAN is a project funded by the Future and Emerging Technologies arm of the IST Programme of the the European Commission. This project investigates the potentialities of the Mobile Ad hoc NETworks (MANET's) paradigm. Specifically, the project aims at defining and developing a metropolitan area, self-organizing, and totally wireless network, called Mobile Metropolitan Ad hoc Network (MobileMAN). The main technical output of this proposal can be summarized as follow. i) Development, validation, implementation and testing of the architecture, and related protocols, for configuring and managing a MobileMAN. ii) Physical implementation of this architecture for lowers layers (i.e., wireless technologies). iii) Integration of applications on top of our self-organized network. iv) Validation of the self-organizing paradigm from the social and economic standpoint.
\end{abstract}

\section{Introduction}

The future information society is expected to rely heavily on wireless technology. Advances in wireless communication will enable a radical new communication paradigm based on self-organized information.

This new networking environment is composed by a group of wireless devices, called nodes, that must cooperatively realize the functionalities usually provided by the network infrastructure (e.g. routing, forwarding, network management). Such systems are known as mobile ad hoc networks [1, or infrastructureless networks. The Internet Engineering Task Force community refers to them as MANETs 2]. The MobileMAN project 3] will advance the MANET paradigm focusing on communications in a (possibly large) metropolitan area, called Mobile Metropolitan Ad Hoc Network (MobileMAN). A MobileMAN is an autonomous, self-organized, wireless network, solely composed by wireless nodes like, for example, next generation mobile phones, emerging Personal Digital Assistants (PDAs), and laptops equipped with wireless interfaces.

* This work was partially funded by the Information Society Technologies programme of the European Commission, Future and Emerging Technologies under the IST2001-38113 MOBILEMAN project. 
Table 1. Project partners

\begin{tabular}{l|l|l} 
Partner & Country & Research areas \\
\hline IIT-CNR & Italy & Wi-Fi, Networking, Transport, Middleware \\
\hline EURECOM & France & Cooperation, Security \\
\hline UCAM-CLAB & United Kingdom & Middleware, Economic Issues \\
\hline HUT & Finland & Networking \\
\hline NETikos & Italy & Applications \\
\hline SUPSI & Switzerland & Wi-Fi, Cooperation, Social Aspects \\
\hline
\end{tabular}

In a MobileMAN the lack of centralized points leads to the necessity of distributing all the network functions to the available nodes, that must cooperate and provide services to each other. While adjacent nodes communicate directly, far away nodes rely on intermediate nodes acting as relays.

A MobileMAN is not intended as a replacement for current infrastructure based (wired and wireless) networks. It is intended to complement them, and to enable new application scenarios in which a centralized infrastructure is impossible, undesirable, or unnecessary. In addition, we also envisage intercommunication between a MobileMAN and the Internet. This can be provided by nodes which have simultaneous access to the MobileMAN and to the Internet, perhaps via special access points, or via user terminals with multiple network interfaces (e.g., a PDA equipped with both Bluetooth and GPRS interfaces). Applications supported by a MobileMAN might range from text messaging systems (evolving from the very popular SMS), up to more demanding multimedia (voice and video) services. Eventually, they might even range from simple interactive games to ones that truly merge real, and virtual worlds.

The project objectives are twofold. On the technical aspect, the project aims at inventing and demonstrating solutions that make the self-organisation paradigm effective. On the business and social aspect, it aims at exploiting the self-organisation paradigm for supporting innovative applications, which will improve people's living standards.

The main expected technical output of MobileMAN regards the development, validation, implementation, and testing of the MobileMAN architecture. Another expected result is the integration and validation of popular services (such as SMS and chatting) on top of our self-organised network, as well as, the extensions of these services into new realms, such as multimedia messaging, spontaneous electronic collaboration, and wireless interactive games. Finally, a socio-economic evaluation of the effectiveness of the mobile ad hoc paradigm, and its market access will be provided.

Table 1 lists all the project participants, with the associated main research areas within the MobileMAN project framework. 


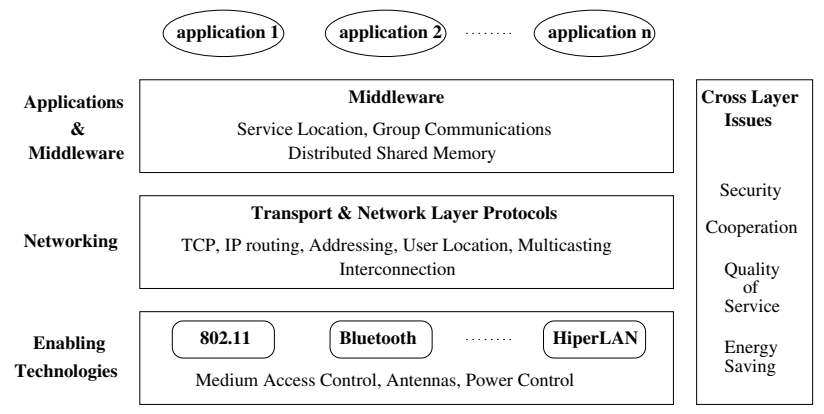

Fig. 1. MANET reference architecture.

\section{MANET Architecture}

Classical networking architectures layer protocols and network responsibilities. Even the MANET architecture groups the ad hoc networking activities into three main areas: enabling technologies, networking, middleware and applications (see Figure 1). This layered approach leads the research efforts to target mainly isolated components of the overall network design (e.g., routing, MAC, power control). In this model, each layer in the protocol stack is designed and operated separately, with interfaces between layers that are static and independent of the individual network constraints and applications. However, as shown in Figure 1] in a MANET some functionalities cannot be assigned to a single layer. For example, energy management, security and cooperation, quality of service, cannot be completely implemented in a single layer, but they are implemented by combining and exploiting mechanisms of all layers.

The layered approach has proved to be effective in wired networks where the conditions are stable. In this case, it guarantees for example that the TCP/IP protocol stack can operate on a large set of heterogeneous technologies. This principle does not apply anymore when wireless technologies are included in the Internet world (see the problems related to TCP over wireless links [4]) and becomes less efficient when ad hoc networks are taken into consideration. Without any doubt, efficient solutions for ad hoc networks require a more strict cooperation among protocols belonging to different layers.

Specifically, we envisage a complete loop in the design/behavior of protocols belonging to different layers. Low layer protocols will be designed taking into account the requirements/characteristics of high-layer protocols. In addition, high layer protocols will react to signalling generated by low layer protocols, as well as low layer protocols will adapt their behavior according to the information collected above them. We think that, in ad hoc networks, efficient solutions can be designed by further strengthening cross-layer interdependencies. For example, adopting multi-path forwarding, TCP acknowledgements can be used to estimate the reliability of different paths. In the following we present the cross-layered MobileMAN architecture. 


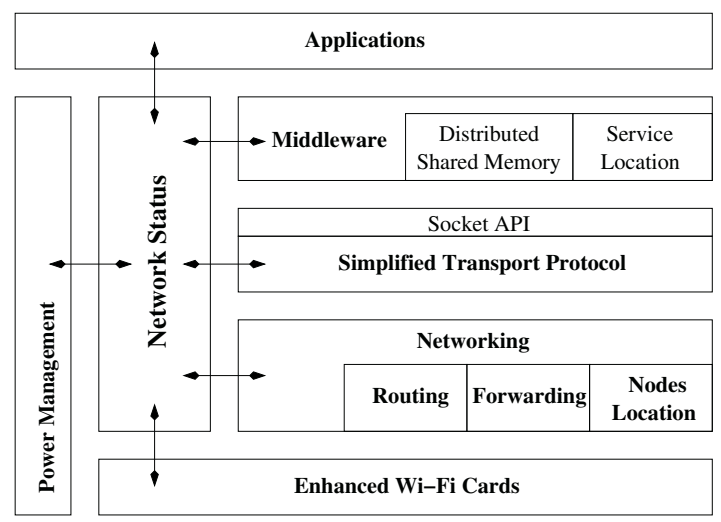

Fig. 2. MobileMAN architecture.

\section{Cross-Layered MobileMAN Architecture}

Information sharing avoids duplicate efforts for collecting data about the network status. For example, in [5] a strict cross-layer design principle is applied to the routing and the middleware layers, to facilitate multimedia data accessibility for various applications at the end-systems. Active communication between the layers is achieved through signalling, regarding the condition of data transmission.

A cross-layer protocol design supports adaptivity across multiple layers of the protocol stack. The different timescales of the network variations suggest that each layer should attempt to compensate for variation at that level. If adapting locally is unsuccessful, information should be exchanged with other layers for a more general response. For example, let us assume that a weak link occurs on a multi-hop path. Firstly, the physical link protocol can try to fix the problem by increasing the transmission power. However, if the link weakness is caused by some physical obstacle, it is better for a higher layer to respond by, for example, delaying packet transmissions (e.g., until the mobile node leaves the tunnel) or by identifying an alternative route. Similarly, if nodes in the network are highly mobile, link characteristics and network topology will change rapidly. Informing the network layer of highly mobile nodes might change the routing strategy from unicast to broadcast in the direction of the intended user. This integrated approach to adaptive networking is the biggest challenge in adaptive protocol design. The above examples support a cross layering design, as it is emerging in the framework of the MobileMAN project [6. The kernel of a MobileMAN network node (see Figure 22) is a shared memory, called Network Status that is a repository of information collected by network protocols at all layers. Each protocol can access this shared memory to read and write collected information. Hence, layers efforts in duplicating network-status information are minimized, facilitating inter-layer cooperation. 
Hereafter we discuss the protocol stack for the ad hoc node, with special attention to exploiting cross-layer interactions.

Wi-Fi. A physical implementation of an enhanced IEEE 802.11 wireless technology will be done by improving the existing standard, dealing with the multi-hop organization of the MobileMAN network [7]. Specifically, the crossleyering functionality will be exploited importing a 2-3 hops horizon knowledge of the neighborhood, built up at the routing layer. This will allow a fair channel scheduling that could take into account the hidden and exposed node issues.

Routing, forwarding, and nodes location. Our claim is to consider the routing problem according to the cross-layering principle, so that routing data is useful also at other layers. For example, the knowledge of node locations can be useful for identifying the closer node that is implementing a service, without requiring the middleware to discover the same location once more. From these observations, our reference choice for routing is based on a link-state protocol. According to the Fisheye policy [8], a node propagates link state information to other nodes in the network, basing on how far away (in terms of hops number) they are. Closer nodes will exchange link state information more frequently than far away nodes. The primary consequence of this choice is that a node has a precise view of the $2-3$ hops network around it, and each node has the knowledge of all the possible paths to a destination. These features help in implementing mechanisms of multi-path reliable forwarding [9], and of security/cooperation enforcing [101112 13]. Considering a possibly large MobileMAN network, the 2-3 hops network knowledge mentioned above is not sufficient to route packets between far away nodes. In this case, a node location service [14] with a geographic forwarding procedure become necessary.

Simplified Transport Protocol. The main goals in the design of a Simplified Transport Protocol, are to provide a reliable and connection-oriented service that minimizes useless data re-transmissions by analysing and reacting appropriately to the different phenomena happening at the below layers (e.g., route failures, route changes, congestions) [15. For this purpose, the Simplified Transport Protocol could exploit information reported by the routing and Wi-Fi layers in the Network Status.

Middleware. The middleware layer generally provides context abstractions, able to hide complex details to application programmers. In mobile ad hoc environments, this trend has to be reversed to context-awareness. The MobileMAN cross-layered architecture aims at supporting this aspect. Finally, recent proposals 17,18, emphasise data sharing among nodes and support for disconnected operations. To achieve this, concepts like tuple spaces, mobile agents, and event based communications have been adopted. While these technologies provide advanced abstractions, efficient implementations are still open issues. Specifically, solutions must be devised to support a distributed shared memory, service discovery, and group communication. 


\section{References}

1. I. Chlamtac, M. Conti, and J. Liu. Mobile Ad Hoc Networking: Imperatives and Challenges. In Ad Hoc Networks, No. 1(1) 2003.

2. http://www.ietf.org/html.charters/manet-charter.html

3. http://cnd.iit.cnr.it/mobileMAN

4. H. Elaarag. Improving TCP Performance over Mobile Networks. ACM Computing Surveys, Vol. 34, No. 3, pp. 357-374, Sept. 2002.

5. K. Chen, S.H. Shah, and K. Nahrstedt. Cross-Layer Design for Data Accessibility in Mobile Ad Hoc Networks. In Wireless Personal Communications No. 21, pp. 49-76, 2002.

6. M. Conti. Cross Layering in MANETs' Design: the MOBILEMAN Approach. The first NSF - COST Workshop on Networking, Chania, Crete, June 2003.

7. R. Bernasconi, I. Defilippis, S. Giordano, and A. Puiatti. An enhanced MAC architecture for multi-hop wireless networks. To appear in Proceedings of the Personal Wireless Communications Conference 2003 (PWC 2003), Sept. 2003.

8. G. Pei, M. Gerla, and T.W. Chen. Fisheye State Routing in Mobile Ad Hoc Networks. In Proceedings of the 2000 ICDCS Workshops, Taipei, Taiwan, Apr. 2000.

9. M. Conti, E. Gregori, and G. Maselli. Towards Reliable Forwarding for Ad Hoc Networks. To appear in Proceedings of the Personal Wireless Communications Conference 2003 (PWC 2003), Sept. 2003.

10. S. Giordano, and A. Urpi. Self-Organized and Cooperative Ad Hoc Networking. In Mobile Ad Hoc Networking, S. Basagni, M. Conti, S. Giordano, I. Stojmenovic (Editors), IEEE Press and John Wiley and Sons, Inc., New York, 2003.

11. P. Michiardi and R. Molva. A game theoretical approach to evaluate cooperation enforcement mechanisms in mobile ad hoc networks. In Proceedings of WiOpt '03, Sophia-Antipolis, March 2003.

12. A. Urpi, M.A. Bonuccelli and S. Giordano. Modelling Cooperation in Mobile Ad Hoc Networks: a Formal Description of Selfishness. In Proceedings of WiOpt '03, Sophia-Antipolis, March 2003.

13. J. Crowcroft, R. Gibbens, F. Kelly and S. Östring. Modelling Incentives for Collaboration in Mobile Ad Hoc Networks. In Proceedings of WiOpt '03, Sophia-Antipolis, March 2003.

14. G. Turi. A Context-Aware Location Service for Metropolitan Ad Hoc Networks. To appear in Proceedings of the Personal Wireless Communications Conference 2003 (PWC 2003), Sept. 2003.

15. G. Anastasi, and A. Passarella. Towards a Novel Transport Protocol for Ad Hoc Networks. To appear in Proceedings of the Personal Wireless Communications Conference 2003 (PWC 2003), Sept. 2003.

16. C. Mascolo, L. Capra, and W. Emmerich. Middleware for Mobile Computing (A Survey). In Networking 2002 Tutorial Papers, editors E. Gregori, G. Anastasi, S. Basagni. Springer. LNCS 2497.

17. Amy L. Murphy, Gian Pietro Picco, and Gruia-Catalin Roman. Lime: A Middleware for Physical and Logical Mobility. In Proceedings of the 21st International Conference on Distributed Computing Systems (ICDCS-21), May 2001.

18. C. Mascolo, L. Capra, S. Zachariadis, and W. Emmerich. XMIDDLE: A DataSharing Middleware for Mobile Computing. Int. Journal on Personal and Wireless Communications. 